\title{
Revelation of the ability of Burkholderia sp. USM (JCM 15050) PHA synthase to polymerize 4-hydroxybutyrate monomer
}

\author{
Nyok-Sean Lau and Kumar Sudesh ${ }^{*}$
}

\begin{abstract}
The nutrition-versatility of Burkholderia sp. strain USM (JCM 15050) has initiated the studies on the use of this bacterium for polyhydroxyalkanoate (PHA) production. To date, the Burkholderia sp. has been reported to synthesize 3-hydroxybutyrate, 3-hydroxyvalerate and 3-hydroxy-4-methylvalerate monomers. In this study, the PHA biosynthetic genes of this strain were successfully cloned and characterized. The PHA biosynthetic cluster of this strain consisted of a PHA synthase (phaC), $\beta$-ketothiolase (phaA), acetoacetyl-CoA reductase (phaB) and PHA synthesis regulator (phaR). The translated products of these genes revealed identities to corresponding proteins of Burkholderia vietnamiensis (99-100 \%) and Cupriavidus necator H16 (63-89\%). Heterologous expression of phaC $C_{B S}$ conferred PHA synthesis to the PHA-negative Cupriavidus necator PHB-4, confirming that phaC $_{B S}$ encoded functionally active protein. PHA synthase activity measurements revealed that the crude extracts of $C$. necator $\mathrm{PHB}^{-} 4$ transformant showed higher synthase activity $(243 \mathrm{U} / \mathrm{g}$ ) compared to that of wild-types Burkholderia sp. (151 U/g) and $\mathrm{C}$. necator H16 (180 U/g). Interestingly, the transformant C. necator PHB-4 harbouring Burkholderia sp. PHA synthase gene accumulated poly(3-hydroxybutyrate-co-4-hydroxybutyrate) with 4-hydroxybutyrate monomer as high as up to 87 mol\% from sodium 4-hydroxybutyrate. The wild type Burkholderia sp. did not have the ability to produce this copolymer.
\end{abstract}

Keywords: Biopolymer, Polyhydroxyalkanoate, PHA synthase, PHA operon, Burkholderia sp.

\section{Introduction}

Since the introduction of phenoformaldehyde plastic in 1909 by Leo Hendrik Baekeland, petrochemical plastics have developed into a major industry and an indispensable commodity for modern life (Meikle, 1995). It is estimated that more than 100 million tonnes of plastics are produced yearly. Most of these plastics end up after their useful life as discarded waste and some are disposed into the marine environment which pose a threat to the aquatic wildlife. In recent years, the widespread and increasing use of petrochemical plastics has raised concerns about the adverse impact of these recalcitrant plastics on the environment. Hence, biobased and biodegradable polymers are gaining widespread interest and acceptance as an alternative to some synthetic plastics. Polyhydroxyalkanoate (PHA), a storage and reserve

\footnotetext{
* Correspondence: ksudesh@usm.my

Ecobiomaterial Research Laboratory, School of Biological Sciences, Universiti Sains Malaysia, Penang 11800, Malaysia
}

compound accumulated naturally in the cytoplasm of numerous bacteria, is being considered as one of the most attractive and promising biodegradable thermoplastics for various industrial and biomedical applications (Sudesh and Iwata, 2008).

Poly(3-hydroxybutyrate) [P(3HB)], the most common type of PHA, has mechanical properties such as Young's modulus and tensile strength similar to polypropylene. Nevertheless, $\mathrm{P}(3 \mathrm{HB})$ is a stiffer and more brittle plastic material compared to polypropylene (Tsuge, 2002). Many other types of PHA with improved mechanical properties are synthesized by incorporating co-monomers such as 3hydroxyvalerate, 3-hydroxyhexanoate and 4-hydroxybutyrate. The underlying challenge for the commercialization of PHA is the higher production cost compared to petrochemical plastics. To achieve the commercial application and wide use of PHA, efforts are directed on effectively lowering the production cost of PHA. Much research has been focused on reducing the production cost by strain 
development, developing more efficient fermentation and recovery processes and using inexpensive carbon sources. At the same time, more PHA applications are being developed, including the high value applications such as medical and pharmaceutical field at which cost of production is not the main concern (Chen, 2009).

Structural analyses in recent years revealed that different types of granule-associated proteins are located on the surface of PHA granules. The proteins include PHA synthases, intracellular PHA depolymerases, phasins, PHA synthesis regulator proteins and etc. (Pötter and Steinbüchel, 2006; Rehm, 2006; Jendrossek, 2009). PHA synthases are the most important enzymes involved in PHA biosynthesis. They can be grouped into four classes based on their in vivo substrate specificities, primary amino acid sequences and subunit composition (Rehm, 2007). Class I synthases, which are represented by the PHA synthase from Cupriavidus necator, are active towards short-chain length $(R)$-hydroxyacyl-CoA consisting of three to five carbon atoms (Pötter and Steinbüchel, 2006). Class II synthases are active towards medium-chain length $(R)$-3-hydroxyacyl-CoA that contain six to fourteen carbon atoms and are represented by Pseudomonas aeruginosa. Class I and II PHA synthases comprise enzymes consisting of only one type of subunit (PhaC) with molecular masses $(\mathrm{Mw})$ between 61 and $68 \mathrm{kDa}$. Class III PHA synthases, represented by the Allochromatium vinosum PHA synthases, comprise two subunits: PhaC and PhaE. Class IV PHA synthases, represented by the enzyme of Bacillus megaterium, consist of two different types of subunits (PhaC and PhaR). Both class III and IV PHA synthases prefer short-chain length $(R)$-hydroxyacyl-CoA. (Rehm, 2003).

Burkholderia sp. USM (JCM 15050) was isolated from oil-polluted wastewater and has been known to utilize various carbon sources e.g. sugars, organic acids and triglycerides for PHA production (Chee et al. 2010; Lau et al. 2010). The focus of this research was the PHA biosynthetic genes cloned from this bacterium. Here, we reported for the first time that the heterologous expression of Burkholderia sp. PHA synthase in C. necator $\mathrm{PHB}^{-} 4$ lead to the accumulation of poly(3-hydroxybutyrate-co-4-hydroxybutyrate), $\mathrm{P}(3 \mathrm{HB}-c o-4 \mathrm{HB})$ with high 4-hydroxybutyrate $(4 \mathrm{HB})$ composition. Although the accumulation of PHA containing $4 \mathrm{HB}$ monomer was not observed in wild-type Burkholderia sp. culture, the PHA synthase of Burkholderia sp. was able to polymerize the 4HB monomer.

\section{Materials and methods}

\section{Bacterial strains, plasmids and media}

All bacterial strains and plasmids used in this study are listed in Table 1. C. necator $\mathrm{PHB}^{-} 4$ was cultivated in nutrient rich (NR) medium containing meat extract (10 g/
$\mathrm{L})$, peptone $(10 \mathrm{~g} / \mathrm{L})$ and yeast extract $(2 \mathrm{~g} / \mathrm{L})$ at $30^{\circ} \mathrm{C}$. Escherichia coli strains were grown at $37^{\circ} \mathrm{C}$ in Lysogeny Broth (LB) medium consisting of the following components (per L): $10 \mathrm{~g}$ casein enzyme hydrolysate, $5 \mathrm{~g}$ yeast extract and $10 \mathrm{~g} \mathrm{NaCl}$. For maintenance of plasmid, kanamycin $(50 \mu \mathrm{g} / \mathrm{mL})$ or ampicilin $(100 \mu \mathrm{g} / \mathrm{mL})$ were added.

\section{Culture conditions for the synthesis of PHA}

To determine the functional expression of the cloned PHA biosynthetic genes in vivo, PHA biosynthesis was carried out with transformant $C$. necator. Both one-stage cultivation and two-stage cultivation were carried out according to the method described previously (Lau et al., 2010; Lau et al., 2011). For inoculum preparation, transformant C. necator was grown in $50 \mathrm{~mL}$ of $\mathrm{NR}$ at $30^{\circ} \mathrm{C}$, $200 \mathrm{rpm} .3 \%(\mathrm{v} / \mathrm{v})$ of the total volume of the seed culture $\left(\mathrm{OD}_{600 \mathrm{~nm}}=4.5\right)$ were inoculated into nitrogen-limiting mineral salts medium (MM). The MM contained (per liter): $3.32 \mathrm{~g} \mathrm{Na}_{2} \mathrm{HPO}_{4}, 2.80 \mathrm{~g} \mathrm{KH}_{2} \mathrm{PO}_{4}, 0.50 \mathrm{~g} \mathrm{NH}_{4} \mathrm{Cl}$, $0.25 \mathrm{~g} \mathrm{MgSO}_{4} \cdot 7 \mathrm{H}_{2} \mathrm{O}$ and $1 \mathrm{~mL}$ trace elements solution (Doi et al., 1995). The trace element solution consisted of $0.22 \mathrm{~g} \mathrm{CoCl}_{2} \cdot 6 \mathrm{H}_{2} \mathrm{O}, 9.7 \mathrm{~g} \mathrm{FeCl}_{3}, 7.8 \mathrm{~g} \mathrm{CaCl}_{2}, 0.12 \mathrm{~g}$ $\mathrm{NiCl}_{2} \cdot 6 \mathrm{H}_{2} \mathrm{O}, 0.11 \mathrm{~g} \mathrm{CrCl}_{3} \cdot 6 \mathrm{H}_{2} \mathrm{O}, 0.16 \mathrm{~g} \mathrm{CuSO}_{4} \cdot 5 \mathrm{H}_{2} \mathrm{O}$ in one liter $0.1 \mathrm{~N} \mathrm{HCl}$ (Kahar et al., 2004). Different carbon sources (crude palm kernel oil, jatropha oil, fructose, 4methylvaleric acid, sodium propionate, sodium valerate, $\gamma$-butyrolactone and sodium 4-hydroxybutyrate) were tested for their ability to promote PHA synthesis in transformant $C$. necator. The cultures were harvested by centrifugation $\left(6000 \mathrm{~g}, 7 \mathrm{~min}\right.$ and $\left.4^{\circ} \mathrm{C}\right)$ after $48 \mathrm{~h}$ of incubation at $200 \mathrm{rpm}, 30^{\circ} \mathrm{C}$. Cell pellets were washed with hexane to get rid of excess oil-based carbon sources before being washed with distilled water. Cells grown in non-palm oil based carbon sources were washed only with distilled water.

For two-stage cultivation, 3\% (v/v) of the inoculum from transformant $C$. necator culture was transferred into fresh NR broth which was then incubated for additional $24 \mathrm{~h}$. The culture was centrifuged aseptically $\left(6000 \mathrm{~g}, 7 \mathrm{~min}\right.$ and $\left.4^{\circ} \mathrm{C}\right)$ and washed with sterile distilled water before being transferred into nitrogen-free MM. The cultures were incubated at $30^{\circ} \mathrm{C}, 200 \mathrm{rpm}$ for $48 \mathrm{~h}$ before being harvested.

\section{Analytical procedures}

The PHA content and composition were analyzed by gas chromatography (GC) spectrometry. Approximately $25 \mathrm{mg}$ of lyophilized cells were subjected to methanolysis in the presence of $15 \%(\mathrm{v} / \mathrm{v})$ sulphuric acid in methanol. The resulting hydroxyacyl methyl esters were assayed according to the method of Braunegg and coworkers. (Braunegg et al., 1978). 
Table 1 Bacterial strains and plasmids used in this study

\begin{tabular}{|c|c|c|}
\hline Strain or plasmid & Relevant characteristics & Source or reference \\
\hline \multicolumn{3}{|l|}{ Strains } \\
\hline E. coli JM109 & $\begin{array}{l}\text { recA1, endA1, gyrA96, thi, hsdR17, supE44, relA1, } \Delta\left(\text { lac-proAB)/F'[traD36, proAB }{ }^{+}, \text {lacl }^{9} \text {, }\right. \\
\text { lacZDM15] }\end{array}$ & Promega \\
\hline E. coli S17-1 & 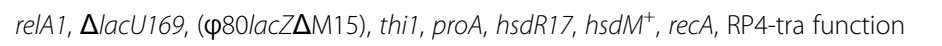 & Simon et al., 1983 \\
\hline C. necator $\mathrm{PHB}^{-4}$ & PHA-negative mutant of wild-type $\mathrm{H} 16$ & Schlegel et al., 1970 \\
\hline Burkholderia sp. USM (JCM 15050) & Wild type & Chee et al., 2010 \\
\hline \multicolumn{3}{|l|}{ Plasmids } \\
\hline pGEM-T & $A p^{r}$, lacZ, cloning vector & Promega \\
\hline pBBR1MCS-2 & $\mathrm{Km}^{r}$, lacPOZ $\mathrm{mob}^{+}$, broad host range & Kovach et al., 1995 \\
\hline pBBR1MCS-2 phaC ${ }_{B S} B H$ & $\begin{array}{l}\text { pBBR1MCS-2 derivative harbouring BamHI-Hindlll phaC from Burkholderia sp. } \\
\text { with putative promoter }\end{array}$ & This study \\
\hline pBBR1MCS-2 phaC ${ }_{B S} B E$ & $\begin{array}{l}\text { pBBR1MCS-2 derivative harbouring BamHI-EcoRl phaC from Burkholderia sp. } \\
\text { with putative promoter }\end{array}$ & This study \\
\hline
\end{tabular}

\section{DNA manipulation}

The isolation of genomic DNA from Burkholderia sp., plasmids DNA isolation, agarose gel electrophoresis and transformation of $E$. coli were performed following standard procedures (Sambrook et al., 1989). Restriction endonucleases, all other DNA-manipulating enzymes and kits were used as recommended by the manufacturers (Promega, USA).

\section{Cloning of PHA biosynthetic genes from Burkholderia sp} The putative PHA synthase $\left(p h a C_{B s}\right), \beta$-ketothiolase $\left(p h a A_{B S}\right)$, acetoacetyl-CoA redutase $\left(p h a B_{B S}\right)$ and PHA synthesis regulator $\left(p h a R_{B S}\right)$ genes were amplified form the chromosomal DNA of Burkholderia sp. using primer pairs I, II and III respectively (Table 2). The resulting polymerase chain reaction (PCR) products were cloned into pGEM-T vector (Promega, USA) and sent for sequencing at 1st BASE Laboratory (Malaysia). Sequence comparisons and alignments were performed with the
Basic Local Alignment Search tool (BLAST, National Center for Biotechnology Information) and ClustalW Multiple Sequence Alignment program (Thompson et al., 1994). The potential promoter regions recognized by Sigma factor $\mathrm{D}\left(\sigma^{\mathrm{D}}\right)$ were predicted using prediction of bacterial promoters (BPROM) provided by Softberry Inc. (http://www.softberry,com).

\section{Construction of plasmids}

To construct a plasmid for expression of $p h a C_{B s}$ in $C$. necator $\mathrm{PHB}^{-} 4, \mathrm{PCR}$ was performed with primer pairs IV to obtain the gene fragment containing the putative coding region, ribosome binding site and promoter. This PCR product was digested with $\mathrm{BamHI}$ and $\mathrm{SacI}$ and inserted in frame into BamHI- and SacI- restricted pBBR1MCS-2. The phaC $C_{B s}$ was also cloned in reverse orientation to lac promoter in pBBR1MCS-2 to confirm the expression under its native promoter. The plasmid for expression was constructed by ligation of $p h a C_{B s}$

Table 2 List of primers used in this study ${ }^{a}$

\begin{tabular}{|c|c|c|}
\hline Primer pairs & Primers' sequences & Target gene \\
\hline \multirow[t]{2}{*}{ । } & F: GCGAGTCACCGAAAATGTITTATGTT & Burkholderia sp. phaC gene \\
\hline & R: TGCGGCCCCTTCAGGTAGTTGTC & \\
\hline \multirow[t]{2}{*}{$\|$} & F: AAGAAGAAGCGCAGCTACTGGGTC & Burkholderia sp. phaA gene \\
\hline & R: TTGAACAGGCTCGTCAGATTGGTG & \\
\hline \multirow[t]{2}{*}{ III } & F: GGCCAGACCAACTATTCGACCGC & Burkholderia sp. phaB and phaR genes \\
\hline & R: GCGACGGCCTTACTTCTTTCCG & \\
\hline \multirow[t]{2}{*}{ IV } & F: GCGAGGATCCGAAAATGTTTTATGTT & Burkholderia sp. phaC gene \\
\hline & R: ATGAGCTCACTACGTCCGTCATTTCC & \\
\hline \multirow[t]{2}{*}{ V } & F: GCGAGGATCCGAAAATGTTTTATGTT & Burkholderia sp. phaC gene \\
\hline & R: ATGAATTCACTACGTCCGTCATTTCC & \\
\hline
\end{tabular}

${ }^{a}$ All primers were synthesized by 1st BASE Laboratory (Malaysia). Restriction enzymes digestion sites were underlined. 
amplified with primer pairs $\mathrm{V}$ into pBBR1MCS-2 at BamHI and EcoRI sites. Conjugation of C. necator $\mathrm{PHB}^{-} 4$ with $E$. coli S17-1 harbouring broad-host-range plasmids were performed as described by Friedrich et al., 1981.

\section{PHA synthase activity assay}

Wild-type Burkholderia sp. and transformant C. necator harbouring pBBR1MCS-2 pha $C_{B S} \mathrm{BH}$ were cultivated for $24 \mathrm{~h}$ under conditions as described in the materials and methods section. Cells were harvested and resuspended in $20 \mathrm{mM}$ Tris- $\mathrm{HCl}(\mathrm{pH}$ 8). Cell extracts were obtained by disruption using sonication (two cycles, $7 \mathrm{~min}$ each) with a TOMY UD-200 sonicator and centrifugation at $13700 \times \mathrm{g}$ for $10 \mathrm{~min}$ at $4^{\circ} \mathrm{C}$. Activities of PHA synthase were determined spectrophotometrically by monitoring the release of $\mathrm{CoA}$ at $412 \mathrm{~nm}\left(30^{\circ} \mathrm{C}\right)$. The standard assay contained $40 \mathrm{mM}$ potassium phosphate buffer $(\mathrm{pH} 7.5)$, $2 \mathrm{mM}$ 3HB-CoA, $10 \mathrm{mM}$ 5,5'-dithio-bis(2-nitrobenzoic acid) and 35 to $40 \mu \mathrm{g}$ of protein from soluble protein fraction (Bhubalan et al. 2011). One unit of enzyme activity was defined as the amount of enzyme that catalyzed the release of $1.0 \mu \mathrm{mol} \mathrm{CoA} / \mathrm{min}$ using a molar absorption coefficient of $13,600 \mathrm{M}^{-1} \mathrm{~cm}^{-1}$. The protein concentration was assayed as described by Bradford using bovine serum albumin as standard.

\section{Results}

Identification and cloning of PHA biosynthetic genes from Burkholderia sp. USM (JCM 15050)

In order to determine the nucleotide sequence of PHA locus of Burkholderia sp. USM (JCM15050), primers for amplification were designed based on the annotated phaC, phaA, phaB and phaR obtained from genomic DNA sequencing of Burkholderia vietnamiensis, Burkholderia thailandensis, Burkholderia ambifaria, Burkholderia glumae, Burkholderia mallei, Burkholderia multivorans and Burkholderia cenocepacia. The nucleotide sequence obtained were deposited with GenBank (accession no. JN022533, JN835296-JN835297, JQ936592). The deduced amino acid sequence of $p h a C_{B s}$ exhibited great similarity to $\mathrm{PhaC}$ of B. vietnamiensis (99\% identity, accession no. YP_001119557.1), Pseudomonas putida (73\% identity, accession no. BAB96552.1) and Cupriavidus necator (63\% identity, accession no. YP_725940.1). The putative ShineDalgarno consensus sequences (TAAGG) were found 10 base pairs upstream of the putative start codon for pha $C_{B s}$. The putative -35 region (TTCACA) and -10 region (TGATAAAAA) found were similar to the corresponding sequences of $E$. coli $\sigma 70$ consensus promoter sequence. The product of $p h a C_{B S}$ is a protein composed of 625 amino acids with a calculated molecular mass of $68.25 \mathrm{kDa}$. Although the nucleotide sequence of phaC from $B$. vietnamiensis was available following complete genomic DNA sequencing, functional characterization of the gene has not been reported. Comparison of the deduced amino acids sequence of phaC from Burkholderia sp. with homologous phaC genes from other bacteria was done by multiple alignment (Figure 1). The deduced amino acids sequence of $p h a C_{B s}$ showed relatively high identity to other PHA synthase and the putative lipase box that has been found conserved in all the PHA synthase was also identified. The deduced amino acid sequences of putative phaA $A_{B s}$ were similar to those $\beta$-ketothiolase from B. vietnamiensis (99\% identity, accession no. YP_001119556.1), P. putida (90\% identity, accession no. BAB96553.1) and C. necator (87\% identity, accession no. YP_725941.1). The putative $p h a B_{B s}$ exhibited significant identity to acetoacetyl-CoA redutase from B. vietnamiensis (99\% identity, accession no. YP_001119555.1), P. putida (92\% identity, accession no. BAB96554.1) and C. necator (89\% identity, accession no. YP_725942.1). A putative PHA synthesis regulator is encoded by $p h a R_{B s}$ and it is located downstream of $p h a B$. The deduced amino acid sequence of phaR exhibited high identity to B. vietnamiensis (100\% identity, accession no. YP_001119554.1) and C. necator (83\% identity, accession no. YP_725943.1). As Burkholderia sp. $16 \mathrm{~S}$ rDNA gene (accession no. FJ667272.1) exhibited 92\% identity to the corresponding gene of $C$. necator (accession no. CP000090.1), it is not surprising to find that the translated products of Burkholderia sp. PHA biosynthetic genes revealed identities to corresponding proteins of $C$. necator (63-89\%).

\section{PHA accumulation in transformant $C$. necator $\mathrm{PHB}^{-} 4$}

The functionality of the cloned Burkholderia sp. USM (JCM 15050) PHA synthase gene was investigated by heterologous expression of the gene in the PHAnegative $C$. necator host. Plasmid pBBR1MCS-2 pha $C_{B S} \mathrm{BH}$ containing $p h a C_{B s}$ and its putative promoter collinear to and downstream of the lac promoter was constructed. The expression was confirmed by complementation of the mutant host deficiency in PHA synthesis and the PHA-producing ability of the transformant strains were examined (Table 3 ). Interestingly, the heterologous expression of phaC $C_{B s}$ in $C$. necator $\mathrm{PHB}^{-} 4$ has conferred the ability to synthesize $\mathrm{P}(3 \mathrm{HB}-\mathrm{co}-4 \mathrm{HB})$ copolymer with high $4 \mathrm{HB}$ composition in the mutant host strain. The $4 \mathrm{HB}$ composition reached $87 \mathrm{~mol} \%$ in cultures supplemented with sodium 4-hydroxybutyrate in two-stage cultivation. In contrast, wild type Burkholderia sp. has limited ability to utilize 4HB-related carbon sources for growth and PHA production (results not shown). The highest PHA content of $66 \mathrm{wt} \%$ accompanied by relatively high dry cell weight of $2.4 \mathrm{~g} / \mathrm{L}$ were obtained in transformant $C$. necator harbouring pha $C_{B s}$ cultivated on crude palm kernel oil (CPKO) in one-stage 


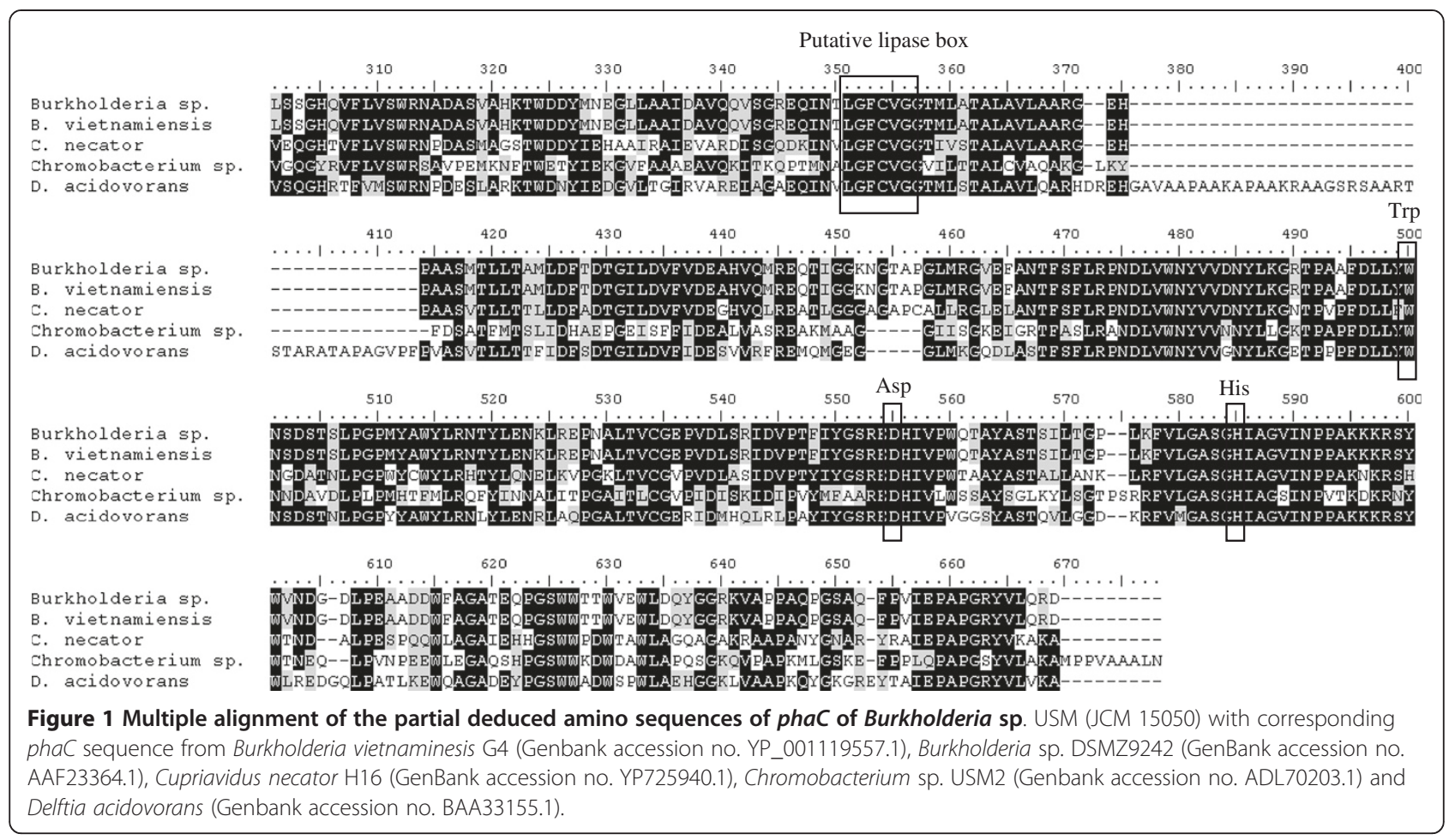

cultivation. Although a low fraction of 3-hydroxy-4methylvalerate (3H4MV) monomer was synthesized by the transformant strain culture from 4-methylvaleric acid, it is in agreement with previous studies on the ability of Burkholderia sp. PhaC to polymerize 3H4MV monomer (Lau et al., 2010). 3-hydroxyvalerate (3HV) monomer was also incorporated into the polymer in the presence of precursor carbon sources e.g. sodium

Table 3 Effect of different carbon sources on the biosynthesis of PHA by transformant Cupriavidus necator PHB`4 harboring the PHA synthase gene of Burkholderia sp.

\begin{tabular}{|c|c|c|c|c|c|c|}
\hline \multirow[t]{2}{*}{ Carbon sources $^{\mathrm{a}}$} & \multirow[t]{2}{*}{ Dry Cell Weight $^{b}$ (g/L) } & \multirow[t]{2}{*}{ PHA content ${ }^{c}$ (wt \%) } & \multicolumn{4}{|c|}{ PHA composition ${ }^{\text {d }}$ (mol \%) } \\
\hline & & & $3 \mathrm{HB}$ & $3 \mathrm{HV}$ & $3 \mathrm{H} 4 \mathrm{MV}$ & 4HB \\
\hline \multicolumn{7}{|l|}{$\underline{\text { One-stage cultivation }}$} \\
\hline \multicolumn{7}{|l|}{ pBBR1MCS-2 phaC $C_{B s} B E$} \\
\hline CPKO & $2.1 \pm 0.4$ & $64 \pm 9$ & 100 & 0 & 0 & 0 \\
\hline \multicolumn{7}{|l|}{ pBBR1MCS-2 phaC $C_{B s} \mathrm{BH}$} \\
\hline CPKO & $2.4 \pm 0.1$ & $66 \pm 2$ & 100 & 0 & 0 & 0 \\
\hline Jatropha oil & $2.3 \pm 0.1$ & $53 \pm 1$ & 100 & 0 & 0 & 0 \\
\hline Fructose & $2.5 \pm 0.1$ & $61 \pm 1$ & 100 & 0 & 0 & 0 \\
\hline Fructose+ 4MV & $1.6 \pm 0.2$ & $40 \pm 8$ & 97 & 0 & 3 & 0 \\
\hline \multicolumn{7}{|l|}{ Two-stage cultivation } \\
\hline Sodium propionate & $2.7 \pm 0.1$ & $27 \pm 3$ & 70 & 30 & 0 & 0 \\
\hline Sodium valerate & $3.2 \pm 0.1$ & $35 \pm 1$ & 60 & 40 & 0 & 0 \\
\hline y-butyrolactone & $2.3 \pm 0.1$ & $29 \pm 1$ & 69 & 0 & 0 & 31 \\
\hline sodium 4-hydroxybutyrate & $2.0 \pm 0.1$ & $14 \pm 2$ & 13 & 0 & 0 & 87 \\
\hline
\end{tabular}

CPKO, crude palm kernel oil; 4MV, 4-methylvaleric acid; 3HB, 3-hydroxybutyrate; 3HV, 3-hydroxyvalerate 3H4MV, 3-hydroxy-4-methylvalerate; 4HB, 4hydroxybutyrate.

${ }^{a}$ Cells were cultivated for $48 \mathrm{~h}$, at $30{ }^{\circ} \mathrm{C}$, $200 \mathrm{rpm}$ in MM medium containing the indicated carbon sources $(0.2 \mathrm{M}$ carbon concentration: CPKO, jatropha oil, fructose, sodium propionate, sodium valerate, $\gamma$-butyrolactone, sodium 4-hydroxybutyrate and $0.02 \mathrm{M}$ carbon concentration: 4-methylvaleric acid). ${ }^{\mathrm{b}}$ Dry cell weight after freeze-drying.

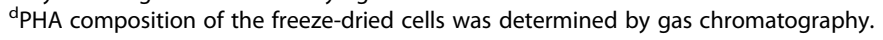


valerate and sodium propionate. To examine whether the expression of $p h a C_{B S}$ was affected by the lac promoter, pBBR1MCS-2 pha $C_{B s} \mathrm{BE}$ was constructed by inserting $p h a C_{B s}$ in reverse orientation to lac promoter. Transformant $C$. necator $\mathrm{PHB}^{-4}$ harbouring pBBR1MCS-2 pha $C_{B S} \mathrm{BE}$ showed similar cell growth and PHA accumulation to strain harbouring pBBR1MCS-2 pha $C_{B S} \mathrm{BH}$. These results suggest that the Burkholderia sp. PHA synthase gene was constitutively expressed in C. necator $\mathrm{PHB}^{-} 4$ from the native promoter.

The ability of transformant $C$. necator $\mathrm{PHB}^{-} 4$ harbouring Burkholderia sp. PHA synthase gene to incorporate $4 \mathrm{HB}$ monomer into the PHA produced was investigated. To study the effect of 4HB-related carbon sources efficiency and concentration on $4 \mathrm{HB}$ composition of PHA synthesized, shake-flask cultures of transformant $C$. necator were cultivated on medium with different concentrations of $\gamma$-butyrolactone or sodium 4-hydroxybutyrate in two-stage cultivation. The dry cell weight, PHA content and $4 \mathrm{HB}$ composition showed slight increase with the increase of sodium 4-hydroxybutyrate concentration from 0.1 to $0.2 \mathrm{M}$ carbon concentration for transformant $C$. necator cultures (Table 4). A maximum of $87 \mathrm{~mol} \% 4 \mathrm{HB}$ composition was produced by the transformant strain cultivated on medium supplemented with $0.2 \mathrm{M}$ carbon concentration sodium 4-hydroxybutyrate. The increase of sodium 4-hydroxybutyrate concentration from 0.2 to $0.4 \mathrm{M}$ carbon concentration resulted in the decrease of $4 \mathrm{HB}$ composition. On the other hand, increased mol fractions of $4 \mathrm{HB}$ monomers in PHA produced were achieved by increasing $\gamma$-butyrolactone concentrations.

\section{Assay of PHA synthase activity}

In order to determine and compare the expression of Burkholderia sp. PHA synthase gene in C. necator
$\mathrm{PHB}^{-} 4$ and its native host, crude extracts of transformant $C$. necator and wild-type Burkholderia sp. were subjected to PHA synthase assay (Table 5). The crude lysate from Burkholderia sp. exhibited specific activity of $151 \mathrm{U} / \mathrm{mg}$, which was similar and comparable with that from C. necator H16 results published previously (180 $\mathrm{U} / \mathrm{mg}$ ) (Schubert et al., 1988). C. necator $\mathrm{PHB}^{-} 4$ harbouring Burkholderia sp. PHA synthase exerted slightly enhanced in vivo PHA synthase activity compared to wild-type Burkholderia sp.

\section{Discussions}

Bacteria belonging to the genus Burkholderia was first used in 1989 for the production of $\mathrm{P}(3 \mathrm{HB})$ homopolymer from fructose (Ramsay et al., 1989). They are one of the most nutritionally versatile microorganisms that are capable of utilizing a wide range of carbon sources. In this study, the PHA biosynthetic genes from a locally isolated Burkholderia sp. strain USM (JCM 15050) were successfully amplified. The PHA biosynthetic genes of Burkholderia sp. consisted of a PHA synthase (phaC), $\beta$-ketothiolase ( $p h a A)$, acetoacetyl-CoA reductase $(p h a B)$ and PHA synthesis regulator (phaR). The phaC, phaA and phaB seems to be organized in an operon and the structural organization of these genes is closely related to other bacteria harbouring type I PHA synthase e.g. C. necator and Alcaligenes latus (Choi et al., 1998; Rehm and Steinbüchel, 2002) (Figure 2). Although the PHA synthase and other PHA biosynthetic genes are often found clustered in the bacterial genomes, there are some exceptions to these observations. In the genomes of Caulobacter crescentus, Paracoccus denitrificans, Methylobacterium extorquens and Aeromonas caviae, the genes related to PHA biosynthesis are not directly linked to the PHA synthase (Rehm and Steinbüchel, 2002). Some

Table 4 Effect of different 4HB-related carbon sources concentrations on the biosynthesis of PHA by transformant Cupriavidus necator $\mathrm{PHB}^{-4}$ harboring the PHA synthase gene of Burkholderia sp.

\begin{tabular}{|c|c|c|c|c|c|}
\hline \multirow[t]{2}{*}{ Carbon sources $^{a}$} & \multirow[t]{2}{*}{ Carbon concentration (M) } & \multirow[t]{2}{*}{ Dry Cell Weight $^{b}$ (g/L) } & \multirow[t]{2}{*}{ PHA content $^{c}$ (wt \%) } & \multicolumn{2}{|c|}{ PHA composition $^{d}$ (mol \%) } \\
\hline & & & & $3 \mathrm{HB}$ & $4 \mathrm{HB}$ \\
\hline Y-butyrolactone & 0.1 & $1.9 \pm 0.1$ & $10 \pm 1$ & 78 & 22 \\
\hline Y-butyrolactone & 0.2 & $2.3 \pm 0.1$ & $29 \pm 1$ & 69 & 31 \\
\hline Y-butyrolactone & 0.3 & $2.4 \pm 0.2$ & $31 \pm 3$ & 68 & 32 \\
\hline Y-butyrolactone & 0.4 & $2.2 \pm 0.1$ & $24 \pm 1$ & 59 & 41 \\
\hline sodium 4-hydroxybutyrate & 0.1 & $1.5 \pm 0.1$ & Trace & 28 & 72 \\
\hline sodium 4-hydroxybutyrate & 0.2 & $2.0 \pm 0.1$ & $14 \pm 2$ & 13 & 87 \\
\hline sodium 4-hydroxybutyrate & 0.3 & $1.9 \pm 0.1$ & $8 \pm 1$ & 40 & 60 \\
\hline sodium 4-hydroxybutyrate & 0.4 & $2.0 \pm 0.1$ & $11 \pm 2$ & 46 & 54 \\
\hline
\end{tabular}

3HB, 3-hydroxybutyrate; 4HB, 4-hydroxybutyrate.

${ }^{a}$ Cells were cultivated for $48 \mathrm{~h}$, at $30^{\circ} \mathrm{C}, 200 \mathrm{rpm}$ in MM medium containing different concentrations of $4 \mathrm{HB}$-related carbon sources at two-stage cultivation.

${ }^{b}$ Dry cell weight after freeze-drying.

'PHA content of the freeze-dried cells was determined by gas chromatography.

${ }^{\mathrm{d}} \mathrm{PHA}$ composition of the freeze-dried cells was determined by gas chromatography. 
Table 5 Analysis of PHA synthase activity in wild-type Burkholderia sp. and transformant $C$. necator

\begin{tabular}{lcl}
\hline Strain & $\begin{array}{l}\text { PHA synthase sp act } \\
(\mathbf{U / g} \text { of protein) }\end{array}$ & References \\
\hline $\begin{array}{l}\text { Burkholderia sp. USM } \\
\text { (JCM 15050) }\end{array}$ & 151 & This study \\
$\begin{array}{l}\text { C. necator } \mathrm{PHB}^{-4} 4 \\
\left.\text { (pBBR1MCS-2 phaC } \text { ps }_{\text {B }} \mathrm{BH}\right)\end{array}$ & 243 & This study \\
C. necator $\mathrm{H16}$ & 180 & Schubert et al., 1988 \\
\hline
\end{tabular}

a One unit of enzyme activity was defined as the amount of enzyme that catalyzed the release of $1.0 \mu \mathrm{mol} \mathrm{CoA} / \mathrm{min}$.

bacteria e.g. $P$. denitrificans contains other genes related to PHA synthesis (phasin, phaP and phaR) map close to the PHA locus. Both $C$. necator and Burkholderia sp. PHA locus also possess a putative phaR immediately downstream of $p h a B$. The phaR encodes a protein with putative regulatory function in PHA metabolism (York et al. 2002; Stubbe and Tian, 2003; Pötter and Steinbüchel, 2006).

The putative translated product of Burkholderia sp. PHA biosynthetic genes exhibited high homologies to the respective $B$. vietnamiensis and $C$. necator genes. As the most important enzyme involved in PHA biosynthesis, PHA synthase of Burkholderia sp. belongs to Class I that is active on short chain-length $(R)$-hydroxyacyl-CoA with three to five carbon atoms (Rehm and Steinbüchel, 2002; Rehm, 2007). Site-specific mutagenesis analysis of $C$. necator PHA synthase had shown that the conserved cystein-319, aspartate- 480 and histidine508 of the class I synthase are required for enzyme activity. These amino acid residues are conserved in all class I synthases and it was suggested that they are involved in covalent catalysis. In addition, another conserved residue, tryptophan-398 was suggested to function in PHA synthase subunit dimerization by the provision of a hydrophobic surface (Gerngross et al., 1994; Jia et al., 2001). In recent years, PHA synthases with improved substrate specificity, enzyme activity and stability were engineered by various approaches e.g. random mutagenesis, gene shuffling and recombination (Nomura and Taguchi 2007). The introduction of a mutant PHA synthase (Ser325Thr/Gln481Lys) from Pseudomonas sp. 61-

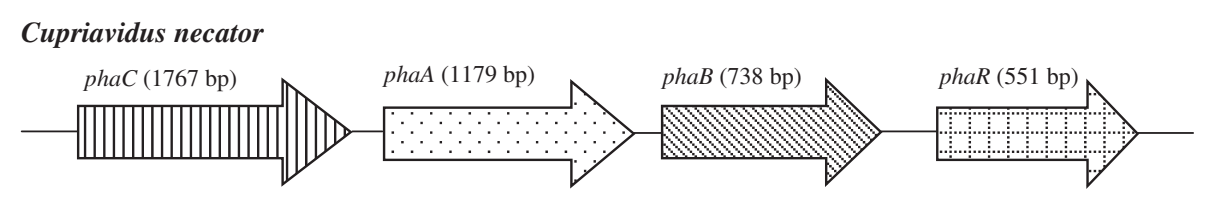

Burkholderia Sp. USM (JCM 15050)

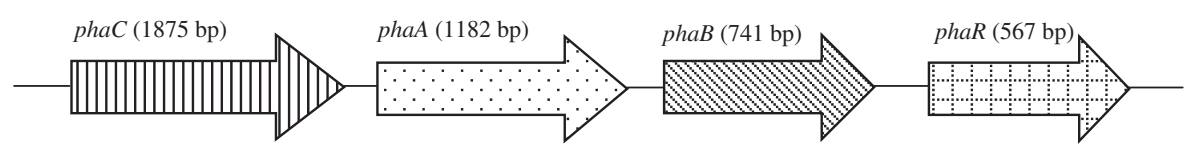

Alcaligenes latus

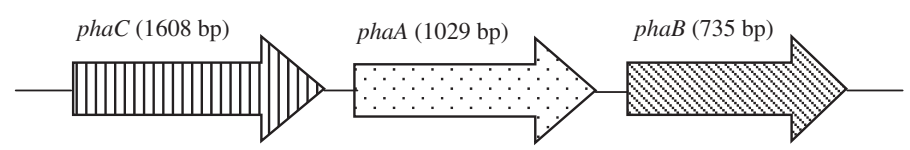

Chromobacterium violaceum

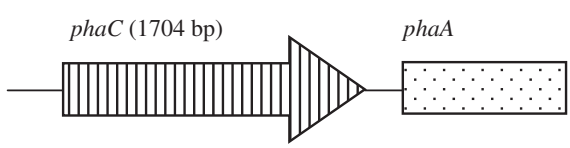

Delftia acidovorans

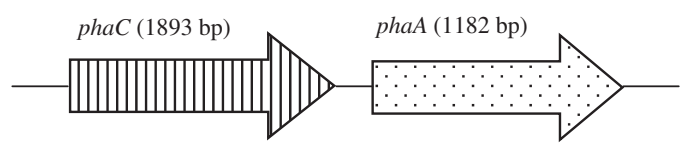

Figure 2 Molecular organizations of PHA biosynthetic genes in Burkholderia sp. USM (JCM 15050) and other bacteria containing type I PHA synthase (Choi et al., 1998; Kolibachuk et al., 1999; Rehm \& Steinbüchel, 2002; Sudesh et al., 1998). 
3 and lactate (LA) monomer supplying enzymes into $E$. coli had enabled the production of LA-based polyesters in a biological system (Shozui et al., 2010). The same mutant Pseudomonas sp. PHA synthase was expressed in E. coli for the synthesis of glycolate-based polyesters containing medium-chain-length 3-hydroxyalkanoates (Matsumoto et al., 2011).

The types and compositions of PHA that are produced by the biological system depend on the PHA synthase substrate specificity, the carbon sources supplied and the metabolic pathways that are functioning in the cell (Sudesh and Doi, 2005). PHA-negative mutant $\mathrm{PHB}^{-} 4$ of C. necator is a desirable host for PHA production due to the strain ability to achieve stable high cell density fermentation and produce high PHA content from simple, inexpensive substrates (Taguchi et al., 2003). Heterologous expression of Burkholderia sp. PHA synthase gene in PHA-negative mutant of $C$. necator enabled the synthesis of $\mathrm{P}(3 \mathrm{HB}-\mathrm{co}-4 \mathrm{HB})$ containing $87 \mathrm{~mol} \%$ of the $4 \mathrm{HB}$ fraction. In contrast, wild-type Burkholderia sp. had reduced ability in utilizing 4HB-related carbon sources for growth and PHA production. It is for the first time that the Burkholderia sp. PHA synthase was shown to polymerize $4 \mathrm{HB}$ monomer. It is well documented that $C$. necator was equipped with the metabolic pathway to supply 4HB-CoA substrate even though only small amount of $\mathrm{P}(4 \mathrm{HB})$ was accumulated (Nakamura et al., 1992). The expression of Burkholderia sp. PHA synthase in a host that are capable of providing 4-hydroxybutyrylCoA as substrate for the PHA synthase allowed the polymerization of $4 \mathrm{HB}$ monomer. These results support previous findings which suggested that the monomer supplying pathways operating in the cells are an important factor determining the provision of substrate for the polymerization by the PHA synthase (Sudesh et al., 1998). In addition, the synthesis of $\mathrm{P}(3 \mathrm{HB}-\mathrm{co}-4 \mathrm{HB})$ with high $4 \mathrm{HB}$ composition by transformant $C$. necator suggested the preference of Burkholderia sp. PHA synthase for 4-hydroxybutyryl-CoA.

In this study, the PHA biosynthetic gene cluster Burkholderia sp. USM (JCM 15050) which consisted of a phaC, phaA, phaB and phaR were successfully cloned. The heterologous expression of Burkholderia sp. PHA synthase gene in $C$. necator $\mathrm{PHB}^{-} 4$ showed that the PHA synthase was capable of polymerizing $4 \mathrm{HB}$ monomer. The biosynthesis results also suggested Burkholderia sp. PHA synthase preference for $4 \mathrm{HB}-\mathrm{CoA}$ as PHA with high $4 \mathrm{HB}$ composition ( $87 \mathrm{~mol} \%$ ) was synthesized by transformant $C$. necator harbouring the synthase. PHA synthase activity assay of wild-type Burkholderia sp. and transformant C. necator indicated that these two strains exhibited activities that are comparable and in a similar range with $C$. necator H16. More efforts to analyze the substrate specificity of PHA synthase in various hosts need to be done in order to understand fully the potential of the synthase.

\section{Competing interests}

The authors declare that they have no competing interests.

\section{Acknowledgements}

We thank Ms. Hor Ling-Hong for her help during the execution of this project. This work was supported by the Exploratory Research Grant Scheme (ERGS) from the Ministry of Higher Education, Malaysia. N. S. Lau thanks Universiti Sains Malaysia Fellowship for the financial support.

Received: 31 July 2012 Accepted: 1 August 2012

Published: 9 August 2012

\section{References}

Bhubalan K, Chuah J-A, Shozui F, Brigham CJ, Taguchi S, Sinskey AJ, Rha C, Sudesh K (2011) Characterization of the highly active polyhydroxyalkanoate synthase of Chromobacterium sp. strain USM2. Appl Environ Microbiol 77:2926-2933

Braunegg G, Sonnleitner B, Lafferty RM (1978) A rapid gas chromatographic method for the determination of poly- $\beta$-hydroxybutyric acid in microbial biomass. Eur J Appl Microbiol 6:29-37

Chee J-Y, Tan Y, Samian M-R, Sudesh K (2010) Isolation and characterization of a Burkholderia sp. USM (JCM15050) capable of producing polyhydroxyalkanoate (PHA) from triglycerides, fatty Acids and glycerols. J Polym Environ 18:584-592

Chen G-Q (2009) A microbial polyhydroxyalkanoates (PHA) based bio- and materials industry. Chem Soc Rev 38:2434-2446

Choi J-I, Lee SY, Han K (1998) Cloning of the Alcaligenes latus polyhydroxyalkanoate biosynthesis genes and use of these genes for enhanced production of poly(3-hydroxybutyrate) in Escherichia coli. Appl Environ Microbiol 64:4897-4903

Doi Y, Kitamura S, Abe H (1995) Microbial synthesis and characterization of poly (3-hydroxybutyrate-co-3-hydroxyhexanoate). Macromol 28:4822-4828

Friedrich B, Hogrefe C, Schlegel HG (1981) Naturally occurring genetic transfer of hydrogen-oxidizing ability between strains of Alcaligenes eutrophus. J Bacteriol 147:198-205

Gerngross TU, Snell KD, Peoples OP, Sinskey AJ, Csuhai E, Masamune S, Stubbe J (1994) Overexpression and purification of the soluble polyhydroxyalkanoate synthase from Alcaligenes eutrophus: evidence for a required posttranslational modification for catalytic activity. Biochemistry 33:9311-9320

Jendrossek D (2009) Polyhydroxyalkanoate granules are complex subcellular organelles (carbonosomes). J Bacteriol 191:3195-3202

Jia Y, Yuan W, Wodzinska J, Park C, Sinskey AJ, Stubbe J (2001) Mechanistic studies on class I polyhydroxybutyrate (PHB) synthase from Ralstonia eutropha: class I and III synthases share a similar catalytic mechanism. Biochemistry 40:1011-1019

Kahar P, Tsuge T, Taguchi K, Doi Y (2004) High yield production of polyhydroxyalkanoates from soybean oil by Ralstonia eutropha and its recombinant strain. Polym Degrad Stabil 83:79-86

Kolibachuk D, Miller A, Dennis D (1999) Cloning, molecular analysis and expression of the polyhydroxyalkanoic acid synthase (phaC) gene from Chromobacterium violaceum. Appl Environ Microbiol 65:3561-3565

Kovach ME, Elzer PH, Hill DS, Robertson GT, Farris MA, Roop RM II, Peterson KM (1995) Four new derivatives of the broad-host-range cloning vector pBBR1MCS, carrying different antibiotic-resistance cassettes. Gene 166:175-176

Lau N-S, Chee J-Y, Tsuge T, Sudesh K (2010) Biosynthesis and mobilization of a novel polyhydroxyalkanoate containing 3-hydroxy-4-methylvalerate monomer produced by Burkholderia sp. USM (JCM15050). Bioresour Technol 101:7916-7923

Lau N-S, Tsuge T, Sudesh K (2011) Formation of new polyhydroxyalkanoate containing 3-hydroxy-4-methylvalerate monomer in Burkholderia sp. Appl Microbiol Biotechnol 89:1599-1609

Matsumoto K, Ishiyama A, Sakai K, Shiba T, Taguchi S (2011) Biosynthesis of glycolate-based polyesters containing medium-chain-length 3hydoxyalkanoates in recombinant Escherichia coli expressing engineered polyhydroxyalkanoate synthase. J Biotechnol 156:214-217

Meikle JL (1995) American plastic: a cultural history. Meikle Rutgers University Press, New Brunswick, N.J 
Nakamura S, Doi Y, Scandola M (1992) Microbial synthesis and characterization of poly(3-hydroxybutyrate-co-4-hydroxybutyrate). Macromolecules 25:4237-4241

Nomura CT, Taguchi S (2007) PHA synthase engineering toward superbiocatalysts for custom-made biopolymers. Appl Microbiol Biotechnol 73:969-979

Pötter M, Steinbüchel A (2006) Biogenesis and structure of polyhydroxyalkanoate granules. In: Shively JM (ed) Inclusions in prokaryotes. Springer-Verlag, Berlin, pp 110-129

Ramsay BA, Ramsay JA, Cooper DG (1989) Production of poly- $\beta$-hydroxyalkanoic acid by Pseudomonas cepacia. Appl Microbiol Biotechnol 55:584-589

Rehm BHA (2003) Polyester synthases: natural catalysts for plastics. Biochem J 376:15-33

Rehm BHA (2006) Genetics and biochemistry of polyhydroxyalkanoate granule self-assembly: the key role of polyester synthases. Biotechnol Lett 28:207-213

Rehm BHA (2007) Biogenesis of microbial polyhydroxyalkanoate granules: a platform technology for the production of tailor-made bioparticles. Curr Iss Mol Biol 9:41-62

Rehm BHA, Steinbüchel A (2002) PHA synthases: the key enzymes of PHA synthesis. In: Doi Y, Steinbüchel A (eds) Biopolymers. Wiley, VCH, Weinheim, pp 173-215

Sambrook J, Fritsch EF, Maniatis T (1989) Molecular cloning: A laboratory manual, 2nd edn. Cold Spring Harbor Laboratory Press, New York

Schlegel HG, Lafferty R, Krauss I (1970) The isolation of mutants not accumulating poly- $\beta$-hydroxybutyric acid. Arch Microbiol 70:283-294

Schubert P, Steinbüchel A, Schlegel HG (1988) Cloning of the Alcaligenes eutrophus genes for synthesis of poly- $\beta$-hydroxybutyric acid (PHB) and synthesis of PHB in Escherichia coli. J Bacteriol 170:5837-5847

Shozui F, Matsumoto K, Nakai T, Yamada M, Taguchi S (2010) Biosynthesis of novel terpolymers poly(lactate-co-3-hydroxybutyrate-co-3-hydroxyvalerate)s in lactate-overproducing mutant Escherichia coli JW0885 by feeding propionate as a precursor of 3-hydroxyvalerate. Appl Microbiol Biotechnol 85:949-954

Simon R, Priefer U, Pühler A (1983) A broad host range mobilization system for in vivo genetic engineering. Transposon mutagenesis in Gram negative bacteria. Bio/Technology 1:784-791

Stubbe J, Tian J (2003) Polyhydroxyalkanoate (PHA) homeostasis: the role of the PHA synthase. Nat Prod Repo 20:445-457

Sudesh K, Doi Y (2005) Polyhydroxyalkanoates. In: Bastioli C (ed) Handbook of biodegradable polymers. Rapra Technology Limited, United Kingdom, pp 219-241

Sudesh K, Iwata T (2008) Sustainability of biobased and biodegradable plastics. CLEAN-Soil, Air, Water 36:433-442

Sudesh K, Fukui T, Doi Y (1998) Genetic analysis of Comamonas acidovorans polyhydroxyalkanoate synthase and factors affecting the incorporation of 4-hydroxybutyrate monomer. Appl Environ Microbiol 64:3437-3443

Taguchi S, Nakamura H, Kichise T, Tsuge T, Yamato I, Doi Y (2003) Production of polyhydroxyalkanoate (PHA) from renewable carbon sources in recombinant Ralstonia eutropha using mutants of original PHA synthase. Biochem Eng J 16:107-113

Thompson JD, Higgins DG, Gibson TJ (1994) CLUSTAL W: improving the sensitivity of progressive multiple sequence alignment through sequence weighting, position-specific gap penalties and weight matrix choice. Nucleic Acid Res 22:4567-4680

Tsuge T (2002) Metabolic improvements and use of inexpensive carbon sources in microbial production of polyhydroxyalkanoates. J Biosci Bioeng 94:579-584

York GM, Stubbe J, Sinskey AJ (2002) The Ralstonia eutropha PhaR protein couples synthesis of the PhaP phasin to the presence of polyhydroxybutyrate in cells and promotes polyhydroxybutyrate production. J Bacteriol 184:59-66

doi:10.1186/2191-0855-2-41

Cite this article as: Lau and Sudesh: Revelation of the ability of Burkholderia sp. USM (JCM 15050) PHA synthase to polymerize 4-hydroxybutyrate monomer. AMB Express 2012 2:41.

\section{Submit your manuscript to a SpringerOpen ${ }^{\circ}$ journal and benefit from:}

- Convenient online submission

- Rigorous peer review

- Immediate publication on acceptance

- Open access: articles freely available online

- High visibility within the field

- Retaining the copyright to your article

Submit your next manuscript at $\gg$ springeropen.com 